WS6-P03

\title{
Surface Wave Analysis - Challenges for Application on an Ultra-shallow Structure Characterisation
}

\author{
C.A. Pérez Solano* (MINES ParisTech), D. Donno (MINES ParisTech), H. \\ Chauris (MINES ParisTech) \& G.P. Deidda (University of Cagliari)
}

\section{SUMMARY}

We discuss several difficulties related to the application of Full Waveform Inversion using surface waves in the context of seismic data acquired on an ultra-shallow ground model $(0-10 \mathrm{~m}$ depth $)$. For instance, the choice of an accurate initial velocity model in the presence of very high velocity contrasts is complicated. The presence of surface waves increases local minima problems in the objective function, preventing from convergence towards the global minimum. For improving convergence, we propose to use an alternative functional, the windowed-Amplitude Waveform Inversion, which is similar to dispersion curve analysis but without any picking step. The source wavelet is iteratively estimated during the velocity model inversion. If previous examples have shown that this methodology is applicable in synthetic data sets, it is less obvious in real data sets as discussed here. 


\section{Introduction}

Near-surface characterisation can be achieved by surface wave analysis (Socco et al., 2010). Classical approaches consist of inverting dispersion curves (previously picked from $f-k$ gathers) using local 1D modelling approaches (Haskell, 1953). Lateral variations have been addressed by splitting the seismic record by means of spatial windows followed by inversion of localised dispersion curves considering laterally constrained inversion schemes (Bergamo et al., 2012).

We propose to invert surface waves using Full Waveform Inversion (FWI). Since it is well-known that FWI might be difficult to use with surface waves, we propose a new FWI-based approach: the windowedAmplitude Waveform Inversion (w-AWI). Synthetic data have been used to demonstrate that w-AWI allows convergence towards the solution even when FWI does not (Perez Solano, 2013). Here, w-AWI is used to provide FWI with an initial Vs model. In this paper, we refer to this strategy as w-AWI \& FWI.

We first describe the two objective functions considered in w-AWI \& FWI. We then present the first real data results obtained by applying this alternative strategy (more details in Pérez Solano (2013)).

\section{Method}

The w-AWI objective function is intermediary between classical FWI and dispersion curve analysis. It is defined as

$$
\Phi_{1}(\mathbf{m})=\frac{1}{2} \sum_{s r c} \sum_{r}\left\|\left|\mathbf{D}_{r}(\mathbf{m})\right|-\left|\mathbf{D}_{r}^{o b s}\right|\right\|^{2},
$$

where $\|.\|^{2}$ defines the $\ell_{2}$ norm, src stands for sources, $|$.$| is the absolute value, the data is computed$ as $\mathbf{D}_{r}(\mathbf{m})=\mathscr{F}_{2 D}\left\{\mathbf{w}_{r}^{T} \mathbf{d}(\mathbf{m})\right\}$ and $\mathbf{D}_{r}^{o b s}=\mathscr{F}_{2 D}\left\{\mathbf{w}_{r}^{T} \mathbf{d}^{o b s}\right\}$, with $\mathscr{F}_{2 D}$ denoting the 2D Fourier transform, $\mathbf{w}_{r}^{T}$ is a sliding window used to split the data before transformation, $r$ is the window position, $\mathbf{d}(\mathbf{m})$ the modelled data and $\mathbf{d}^{\text {obs }}$ the observed data (Pérez Solano, 2013). The classical FWI function is defined as

$$
\Phi_{2}(\mathbf{m})=\frac{1}{2} \sum_{s r c} \mathbf{P}\left\|\mathbf{d}(\mathbf{m})-\mathbf{d}^{o b s}\right\|^{2},
$$

where $\mathbf{P}$ is the data weighting (Virieux and Operto, 2009). In this work, the modelled data $\mathbf{d}(\mathbf{m})$ is computed in the $2 \mathrm{D}$ elastic approximation.

\section{Real data application}

The ultra-shallow ground model consists of a concrete structure buried into a silt and clay background (Deidda and Balia, 2001). The seismic line is perpendicular to the concrete structure and consists of 61 shot gathers with 72 geophones each (geometry in Figure 1, left). Data preprocessing includes amplitude correction, interpolation of edited traces, $t-x$ masking and frequency filtering $(10-70 \mathrm{~Hz})$. First-arrival
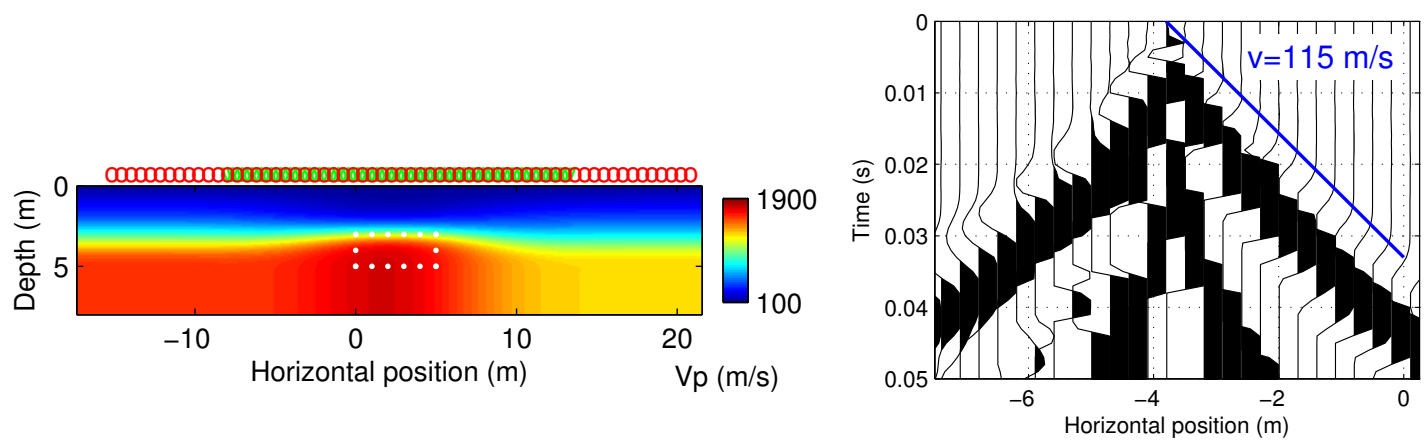

Figure 1 The Vp model obtained by using first-arrival traveltime tomography (left) shows a very-low surface velocity also identified in the data (right). White points indicate the concrete structure location. Source positions are shown in red and receivers in green. 

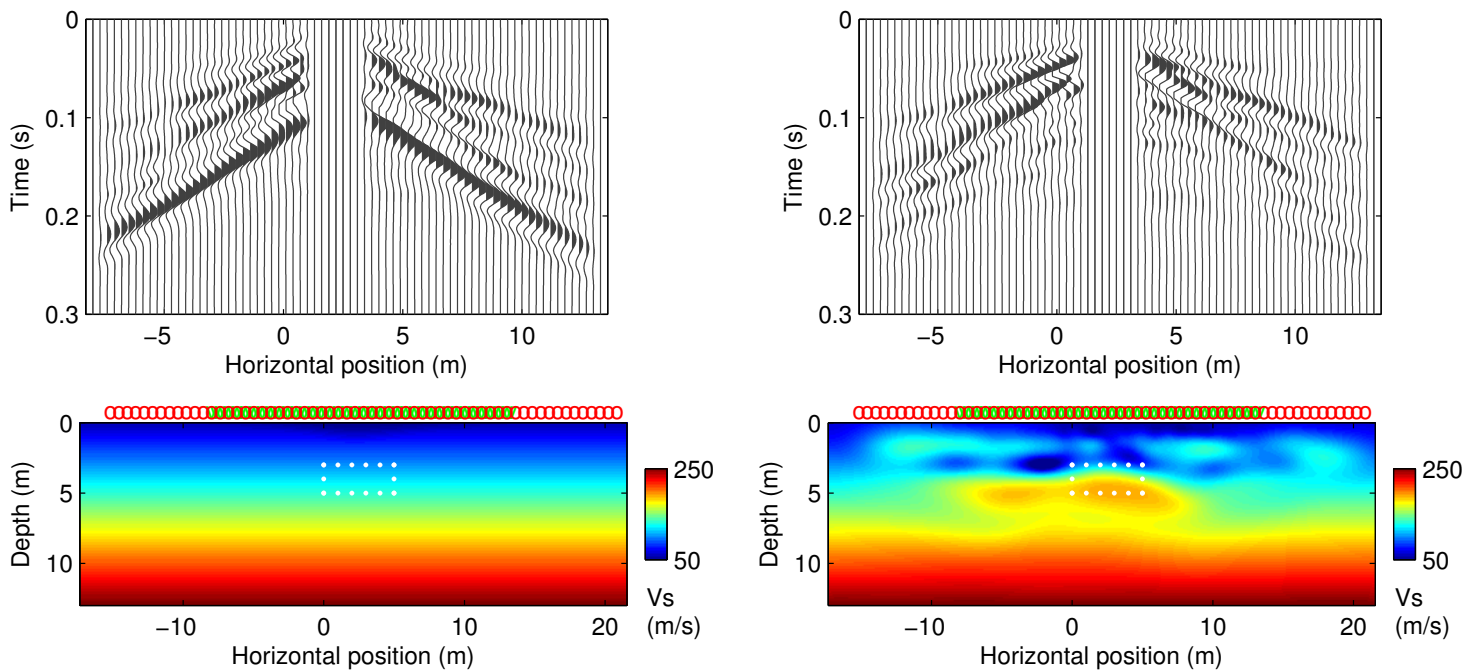

Figure 2 Results of w-AWI \& FWI. Top: initial (left) and final (right) data residuals. Bottom: initial (left) and final (right) Vs model.

traveltime tomography was used to estimate a Vp model (Figure 1, left), allowing for a good retrieval of the top of the concrete structure. This model exhibits a very-low shallow velocity (around $100 \mathrm{~m} / \mathrm{s}$ ) that is confirmed by the slope picked over the data (Figure 1, right). Then, w-AWI \& FWI was applied using a simple linear gradient as initial Vs model. The shot gather residuals show that inversion mostly explained the late arrivals, which are associated to surface waves (Figure 2, top). However, early arrivals still present relatively large residuals, probably because the algorithm ended up in a local minimum. The Vs model obtained by w-AWI \& FWI shows a high-velocity zone close to the position of the concrete structure and the expected velocity inversion below the structure (Figure 2, bottom). There are however some unexpected shallower velocity inversions. One of the causes for this is related to 3D effects originated at the borders of the concrete structure. Besides, interpolation might have masked or destroyed some of the useful data, especially at the negative horizontal positions.

\section{Conclusion}

We have applied a FWI-based inversion strategy, w-AWI \& FWI, to real data in an ultra-shallow ground model. This approach works properly with synthetic data, however with real data we have encountered some difficulties. For instance, the media here contains very-low velocity at the surface that is contrasted with a high-velocity concrete structure. This increases the difficulty to find an accurate starting model for inversion. Nonetheless, the data residuals show that the observed data is fairly well explained, at least for surface waves. This result is encouraging, but the final model can be improved if, for example, an initial Vs model is estimated using dispersion curve analysis prior to w-AWI \& FWI.

\section{References}

Bergamo, P., Boiero, D. and Socco, L.V. [2012] Retrieving 2D structures from surface-wave data by means of spatial-varying spatial windowing. Geophysics, 77, EN39-EN51.

Deidda, G.P. and Balia, R. [2001] An ultrashallow SH-wave seismic reflection experiment on a subsurface ground model. Geophysics, 66, 1097-1104.

Haskell, N.A. [1953] The dispersion of surface waves in multilayered media. Bulletin of the Seismological Society of America, 43, 17-34.

Pérez Solano, C.A. [2013] Two-dimensional near-surface seismic imaging with surface waves: alternative methodology for waveform inversion. PhD thesis. 225 p. MINES ParisTech, France.

Socco, L.V., Foti, S. and Boiero, D. [2010] Surface-wave analysis for building near-surface velocity models Established approaches and new perspectives. Geophysics, 75(5), 75A83-75A102.

Virieux, J. and Operto, S. [2009] An overview of full-waveform inversion in exploration geophysics. Geophysics, 74, WCC1-WCC26. 